\title{
Shelf-Life Prediction of Extra Virgin Olive Oils Using an Empirical Model Based on Standard Quality Tests
}

\author{
Claudia Guillaume and Leandro Ravetti \\ Modern Olives, 151 Broderick Road, Lara, VIC 3212, Australia \\ Correspondence should be addressed to Claudia Guillaume; lab@modernolives.com.au
}

Received 8 July 2016; Accepted 18 August 2016

Academic Editor: Nicola Caporaso

Copyright ( 2016 C. Guillaume and L. Ravetti. This is an open access article distributed under the Creative Commons Attribution License, which permits unrestricted use, distribution, and reproduction in any medium, provided the original work is properly cited.

Extra virgin olive oil shelf-life could be defined as the length of time under normal storage conditions within which no off-flavours or defects are developed and quality parameters such as peroxide value and specific absorbance are retained within accepted limits for this commercial category. Prediction of shelf-life is a desirable goal in the food industry. Even when extra virgin olive oil shelflife should be one of the most important quality markers for extra virgin olive oil, it is not recognised as a legal parameter in most regulations and standards around the world. The proposed empirical formula to be evaluated in the present study is based on common quality tests with known and predictable result changes over time and influenced by different aspects of extra virgin olive oil with a meaningful influence over its shelf-life. The basic quality tests considered in the formula are Rancimat ${ }^{\circledR}$ or induction time (IND); 1,2-diacylglycerols (DAGs); pyropheophytin $a$ (PPP); and free fatty acids (FFA). This paper reports research into the actual shelf-life of commercially packaged extra virgin olive oils versus the predicted shelf-life of those oils determined by analysing the expected deterioration curves for the three basic quality tests detailed above. Based on the proposed model, shelf-life is predicted by choosing the lowest predicted shelf-life of any of those three tests.

\section{Introduction}

Organoleptic and nutritional quality of extra virgin olive oils has been the driving force behind the global growth in their consumption over the past decades. A matter of great significance for producers, retailers, and consumers is undoubtedly the maintenance and assurance of the extra virgin olive oil quality throughout the commercial cycle [1]. The quality of extra virgin olive oil decreases during storage and is attributable to oxidation that leads to rancidity and to hydrolytic degradations causing partial loss of minor components [2]. The characteristic triacylglycerol composition rich in saturated and monounsaturated fatty acids and the presence of natural antioxidants (mainly polar phenols and $\alpha$-tocopherols) are established key factors for the resistance of the oil to autoxidation. A series of other constituents, namely, free fatty acids, pigments, unsaturated hydrocarbons, enzymes, and trace metals, are expected to affect positively or negatively, though to a lesser extent, extra virgin olive oils' stability [3-6].
Extra virgin olive oil shelf-life could be defined as the length of time under normal storage conditions within which no off-flavours or defects are developed and quality parameters such as peroxide value and specific absorbance are retained within accepted limits for this commercial category. Prediction of shelf-life is a desirable goal in the food industry. Even when extra virgin olive oil shelf-life should be one of the most important quality markers for extra virgin olive oil, it is not recognised as a legal parameter in most regulations and standards around the world [7]. In order to estimate extra virgin olive oil resistance to oxidation, the Rancimat or $\mathrm{AOM}$ test results were found meaningful $[8,9]$. It has been shown, nonetheless, that extrapolation from the Rancimat values to ambient conditions leads to either overprediction (most commonly) or underprediction of the actual shelf-life depending on the fatty acid composition of the oils and their antioxidant content [1]. Prediction methods based on kinetic and/or mathematical approaches seem promising but they usually require considerations over the likely temperature of 
storage, light incidence, and packaging material to provide meaningful results [10-13].

In recent from-the-shelf studies of extra virgin olive oils from around the world, consistently more than $50 \%$ of oils sampled tend to fail basic quality parameters for the commercial grade specified on the label. Ageing and/or inappropriate labelling of the best before date are usually pointed out as the two main reasons for such failures that can lead to legal actions for mislabelling and/or bad experiences for consumers [14-16]. The development of an effective tool to predict extra virgin olive oil shelf-life is considered of paramount importance in order to protect consumers and to avoid the commercialisation of oils that do not comply with the regulatory parameters for the commercial grade stated on the label.

Taking all those aspects into consideration, the proposed empirical formula to be evaluated in the present study is based on common quality tests with known and predictable result changes over time and influenced by different aspects of extra virgin olive oil with a meaningful influence over its shelf-life as analysed in previous paragraphs.

\section{Materials and Methods}

2.1. Basic Quality Parameters. Determination of free fatty acids (FFA) (AOCS Ca 5a-40), peroxide value (PV) (AOCS Cd 8-53), and UV coefficients, $K_{232}, K_{270}$, and $\Delta K$ (AOCS Ch 5-91), were carried out. Results were expressed as percentages of oleic acid, milliequivalents $\mathrm{O}_{2} / \mathrm{kg}$ oil, and extinction at 232 and $270 \mathrm{~nm}$, respectively.

2.2. Induction Time (IND). Induction time (AOCS Cd 12b92) was measured with a 743 Rancimat instrument (Metrohm \& Co) using an oil sample of $2.5 \mathrm{~g}$ warmed at $110^{\circ} \mathrm{C}$ and in a $20 \mathrm{~L} / \mathrm{h}$ air flow. The results were expressed in hours. The predicted deterioration curve based on the Rancimat values was calculated at a rate of 720 hours for every hour at $110^{\circ} \mathrm{C}$ as a result of the average evidence shown by previous research [17].

2.3. Pyropheophytin a (PPPs). Pigments were isolated by SPE $\mathrm{SiOH}$ column $6 \mathrm{~mL} / 500 \mathrm{mg}$ (Chromabond Macherey-Nagel $\mathrm{GmbH} \& \mathrm{Co}$.) using acetone as the elution solvent. The eluate was analysed by RP18-HPLC and the separated components were monitored at $410 \mathrm{~nm}$ using a photometric detector. The results were expressed as relative proportions (\%) of the analyses (pheophytin $a$ and pheophytin $a^{\prime}$ and pyropheophytin a). The predicted deterioration curve based on the PPPs values was calculated as the expected time required for the oil to reach a value of $17 \%$ of PPP (PPP limit for extra virgin olive oil according to the Australian Standard AS 5264-2011 and Californian Standard) with predicted monthly increase of $0.6 \%$ as a result of the average evidence shown by previous research [17].

2.4. 1,2-Diacylglycerol Content (DAGs). The isomeric diacylglycerols were isolated by SPE SiOH column $6 \mathrm{~mL} / 500 \mathrm{mg}$ (Chromabond Macherey-Nagel GmbH \& Co.) using diethyl ether as elution solvent. The elute was analysed by gas chromatography after silylation. The peak areas of 1,2- and 1,3-isomers were determined. Only C32, C34, and C36 diacylglycerols were taken into account. The results were expressed as mass percentage (\%) of 1,2-diacylglycerols over the total amount of 1,2- and 1,3-diacylglycerols content in the sample. The predicted deterioration curve based on the DAGs values was calculated as the expected time required for the oil to reach a value of $35 \%$ of DAGs (DAGs limit for extra virgin olive oil according to the Australian Standard AS 5264-2011 and Californian Standard) with predicted monthly decrease of $1.70 \%, 2.10 \%$, or $2.50 \%$ depending on the initial FFA of the oil $(<0.4 \%, 0.4 \%-0.6 \%$, and $>0.6 \%$, resp. $)$ as a result of the average evidence shown by previous research [17].

2.5. Sensory Analysis. Sensory analysis of samples was carried out by trained panellists according to the method described in the International Olive Council (IOC/T.20/Doc. N ${ }^{\circ} 15-\mathrm{Rev}$. 4 November 2011). The method involves, as a measurement, a group of 12 people suitably selected and trained to identify and evaluate the intensities of positive and negative sensory perceptions. Samples were randomly presented and tasters were requested to mark their perceptions on a profile sheet and to evaluate their intensity on an unstructured scale ranked from 0 to 10 . The procedure was repeated three times in different orders to minimise the error. Data provided by tasters were statistically processed to verify the reliability of the test. The median values of the defect (DEF) and positive attributes, fruitiness (FRU); bitterness (BIT); and pungency (PUN) perceived, were utilised to identify the oil category. Samples classified as defective were sent to a second laboratory for confirmation.

2.6. Prediction Model. A wide range of commercial extra virgin olive oils were analysed for induction time, pyropheophytin $a, 1,2$-diacylglycerol content, and free fatty acids prior to their packaging over a period of 30 months and assigned a best before date (BBD) based on the following prediction model.

Best before date is determined by the lowest of the following three estimations:

(i) Hours of induction time at $110^{\circ} \mathrm{C} \times 1=$ expected shelflife (in months).

(ii) $(17.0 \%-\mathrm{PPPs}) / 0.6 \%=$ expected shelf-life (in months).

(iii) $($ DAGs $-35.0 \%) / F F A$ factor $=$ expected shelf-life (in months).

FFA factor $=1.7 \%$ (if FFA $<0.4 \%$ ); $2.1 \%$ (if $0.4 \%<\mathrm{FFA}<0.6 \%$ ); or $2.5 \%$ (if FFA $>0.6 \%$ ).

The evaluation of the prediction model was conducted in two separate ways.

2.7. Evaluation of Prediction Model at the End of Established Shelf-Life. 118 samples packaged during the 30 -month period were analysed for basic quality parameters and organoleptic assessment at the end of their established shelf-life. In order to do that, retention samples were stored under controlled conditions (dark environment and stable temperature at 
TABLE 1: Summary of the complete list of quality analysis performed at the end of estimated shelf-life (118 samples).

\begin{tabular}{lcccc}
\hline Parameter Number of samples failing AS 5264-2011 & Percentage of samples failing AS 5264-2011 & Average & Standard deviation & Median \\
\hline FFA & 1 & $0.8 \%$ & 0.4 & 0.1 \\
PV & 0 & $0.0 \%$ & 7.6 & 1.8 \\
$K_{232}$ & 0 & $0.0 \%$ & 1.747 & 0.170 \\
$K_{270}$ & 2 & $1.7 \%$ & 0.141 & 0.028 \\
$\Delta K$ & 0 & $0.0 \%$ & 0.001 & 0.001 \\
PPP & 12 & $10.2 \%$ & 13.9 & 2.4 \\
DAGs & 6 & $5.1 \%$ & 44.6 & 0.136 \\
Defects & 10 & $8.5 \%$ & 0.1 & 0.001 \\
Fruitiness & 0 & $0.0 \%$ & 3.9 & 0.3 \\
Bitterness & N/A & N/A & 13.8 & 0.5 \\
Pungency & N/A & N/A & 2.5 & 0.0 \\
Failure & 22 & $18.6 \%$ & 4.0 & 0.5 \\
\hline
\end{tabular}

N/A: not applicable.

$18^{\circ} \mathrm{C} \pm 2^{\circ} \mathrm{C}$ ) and tested immediately after reaching their BBD. The 118 samples considered in the evaluation of the prediction model at the end of established shelf-life included $9250 \mathrm{~mL}$ dark glass bottles, $22375 \mathrm{~mL}$-dark glass bottles, $22500 \mathrm{~mL}$ dark glass bottles, $27750 \mathrm{~mL}$-dark glass bottles, 6 one-litre dark glass bottles, 22 3-litre metallic tins, and 10 4-litre metallic tins. Shelf-life predictions ranged from 9 months to 28 months.

2.8. Evaluation of Prediction Model Directly from Retail Points of Sale. 20 samples of the oils packaged with predicted shelflife were randomly collected from different retail points of sale on a quarterly basis during a 30-month period (200 samples in total). Oils were analysed for basic quality parameters and organoleptic assessment. A new BBD based on these quality parameters was recalculated and compared against the previously established BBD.

\section{Results and Discussion}

The basic quality tests considered in the formula are induction time (IND); 1,2-diacylglycerols (DAGs); pyropheophytin $a$ (PPP); and free fatty acids (FFA). The induction time test provides strong correlation of results over time depending on the fatty acid profile of the oils and their antioxidant content. Those factors are well known to be influenced by the variety, environmental conditions, and management practices. The PPP test provides strong correlation over time with light exposure and storage temperature without being influenced by oil quality or the oil's chemical composition. Finally, the DAGs test provides strong correlation over time with temperature of storage and initial oil quality expressed through its FFA content. As it can be seen, the three basic quality tests chosen for the prediction formula are influenced by different factors in the evolution of their values over time.

3.1. Evaluation of Prediction Model at the End of Established Shelf-Life. Table 1 shows the summary of all quality and organoleptic analyses performed on the 118 retention samples with predicted BBDs established during the 30-month period of the trial. Of the 118 samples, only one sample $(0.8 \%$ of the total) marginally exceeded the legal limit of $0.8 \%$ for free fatty acids; no samples failed to comply with either peroxide value or $K_{232}$ limits of $20 \mathrm{meq} \mathrm{O}_{2} / \mathrm{kg}$ and 2.50 , respectively; two samples $\left(1.7 \%\right.$ of the total) failed $K_{270}$ limit of 0.22 ; twelve samples $(10.2 \%)$ failed the AS limit of $17.0 \%$ for pyropheophytin $a$; six samples (5.1\%) failed the AS limit of $35.0 \%$ for 1,2-diacylglycerols; and ten samples (8.5\%) failed their organoleptic assessment for extra virgin olive oil with some form of defect detected.

It is important to highlight that some samples failed multiple tests. Consequently, 22 samples (18.6\% of the total) failed one or more tests for the established legal limits for extra virgin olive oil according to the Australian Standard AS 5264-2011. If we do not consider the results of PPPs and DAGs as per the IOC regulation on olive oils, just 10 samples ( $8.5 \%$ of the total) failed to comply with the established limits for extra virgin olive oil at the end of the predicted shelf-life. This rate of failure is significantly lower than that observed in many other from-the-shelf studies.

Average and median values of PPP and DAGs at the end of the oils' shelf-life as well as their distribution as shown in Figures 1 and 2 indicate that the evolution of those parameters has been reasonably well predicted. 92 retention samples (77.8\% of the total) showed PPP values between $11.0 \%$ and $17.0 \%$ at the end of their shelf-life. 87 retention samples $(73.7 \%$ of the total) showed DAGs values between $35.0 \%$ and $45.0 \%$ at the end of their shelf-life.

3.2. Evaluation of Prediction Model Directly from Retail Points of Sale. During the quarterly random testing of 20 samples over a 30-month period, it was possible to confirm that the average and median values of all quality parameters were well within the established legal limits for extra virgin olive oil (see Tables 2 and 3). Only 3 samples (1.5\%) out of the 200 samples analysed failed to be fully compliant with all legal quality parameters according to AS 5264-2011 and only 1 sample $(0.5 \%)$ out of the 200 samples analysed failed to be 


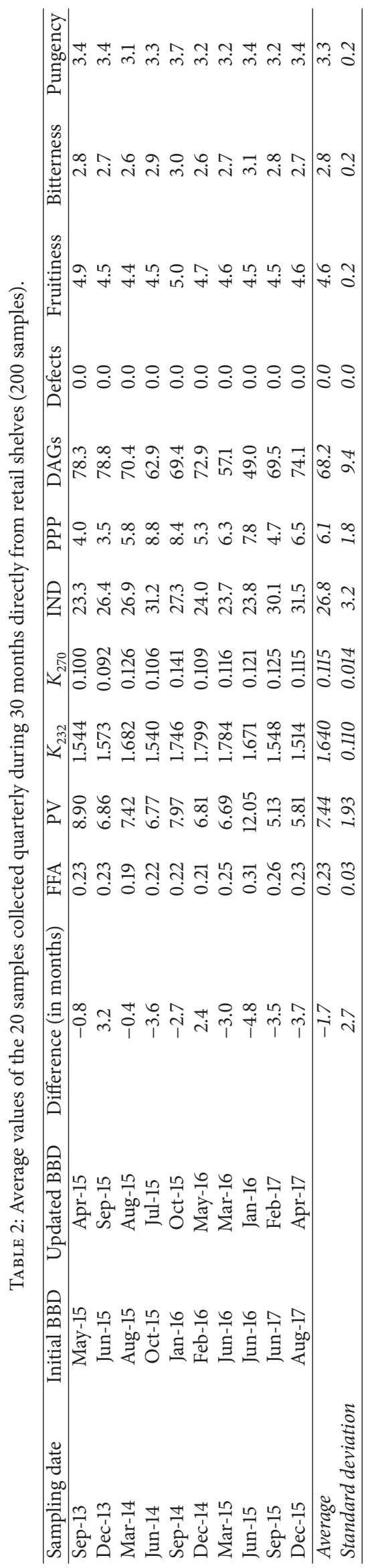




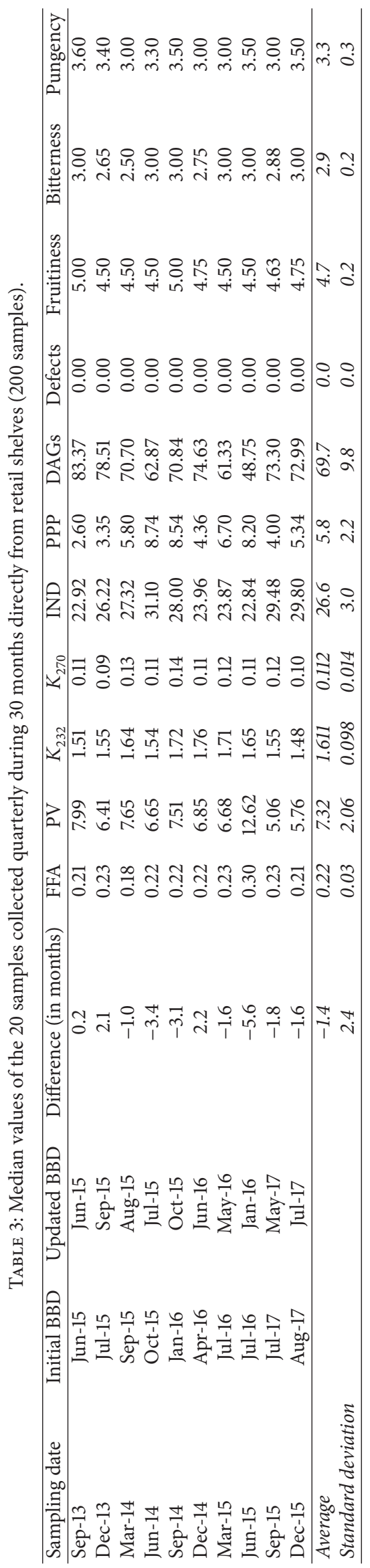


TABLE 4: Number of samples failing AS 5264-2011 limits during the 30 months of retail shelf testing.

\begin{tabular}{lcccccccccccc}
\hline Sampling date & FFA & PV & $K_{232}$ & $K_{270}$ & IND & PPP & DAGs & Defects & Fruitiness & Bitterness & Pungency & Total \\
\hline Sep-13 & 0 & 0 & 0 & 0 & N/A & 0 & 0 & 0 & 0 & N/A & N/A & 0 \\
Dec-13 & 0 & 0 & 0 & 0 & N/A & 0 & 0 & 0 & 0 & N/A & N/A & 0 \\
Mar-14 & 0 & 0 & 0 & 0 & N/A & 0 & 0 & 0 & 0 & N/A & N/A & 0 \\
Jun-14 & 0 & 0 & 0 & 0 & N/A & 0 & 0 & 0 & 0 & N/A & N/A & 0 \\
Sep-14 & 0 & 0 & 0 & 0 & N/A & 0 & 0 & 0 & 0 & N/A & N/A & 0 \\
Dec-14 & 0 & 0 & 0 & 0 & N/A & 0 & 0 & 0 & 0 & N/A & N/A & 0 \\
Mar-15 & 0 & 0 & 0 & 0 & N/A & 0 & 0 & 0 & 0 & N/A & N/A & 0 \\
Jun-15 & 0 & 0 & 0 & 0 & N/A & 0 & 0 & 0 & 0 & N/A & N/A & 0 \\
Sep-15 & 0 & 0 & 0 & 1 & N/A & 0 & 1 & 0 & 0 & N/A & N/A & 2 \\
Dec-15 & 0 & 0 & 0 & 0 & N/A & 0 & 1 & 0 & 0 & N/A & N/A & 1 \\
\hline Total & 0 & 0 & 0 & 1 & 0 & 0 & 2 & 0 & 0 & 0 & 0 \\
\hline
\end{tabular}

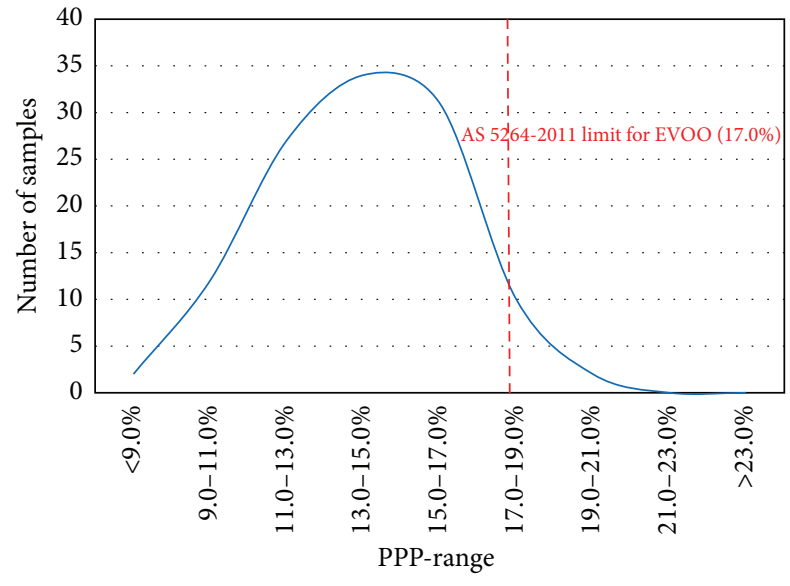

FIGURE 1: Distribution of samples according to PPP results at the end of predicted shelf-life.

fully compliant with all legal quality requirements according to International Olive Council regulations (see Table 4).

When the average and median initially predicted best before dates were compared with the recalculated best before dates based on the quality tests performed on the samples, it was possible to observe that, as an average, the actual samples were showing an expected best before date between 1 and 2 months shorter than what is initially predicted. It is possible that the slightly faster than predicted deterioration was due to the external conditions in the retail shelves and warehouse, such as (a) higher exposure to light, (b) higher than ideal storage temperature during the permanence on the shelves and/or transport, and (c) warehouse stock handling.

\section{Conclusions}

The results of the present study indicate that the shelf-life of extra virgin olive oils can be reasonably predicted by measuring some key quality parameters such as induction time (IND); 1,2-diacylglycerols (DAGs); pyropheophytin $a$ (PPP); and free fatty acids (FFA). These basic quality tests

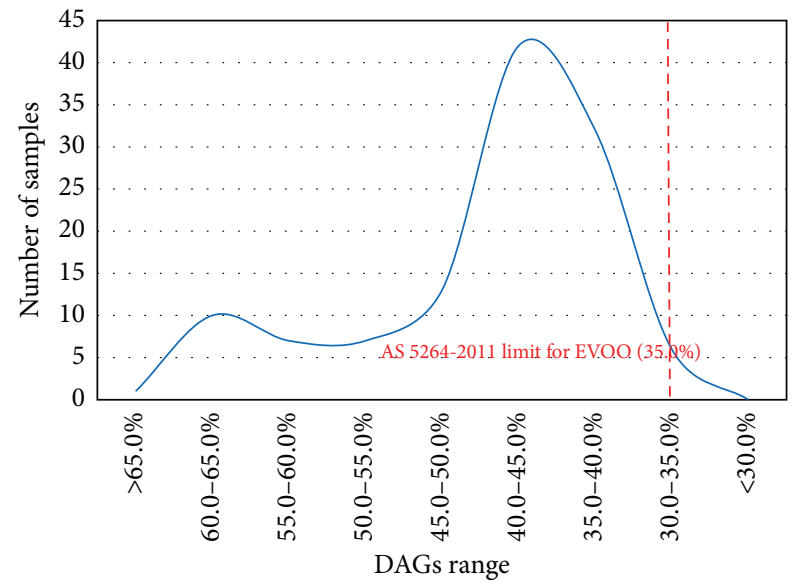

FIGURE 2: Distribution of samples according to DAGs results at the end of predicted shelf-life.

chosen for the prediction formula are influenced by different factors in the evolution of their values over time.

This paper reports research into the actual shelf-life of commercially packaged extra virgin olive oils versus the predicted shelf-life of those oils determined by analysing the expected deterioration curves for the three basic quality tests detailed above. Based on the proposed model, shelf-life is predicted by choosing the lowest predicted shelf-life of any of those three tests.

When evaluated at the end of their predetermined best before date, only a minor percentage of the oils that participated in the study marginally failed one or more chemical and/or organoleptic limits for extra virgin olive oil according to most common international standards. The vast majority of oils displayed quality values similar to those predicted in the model falling just short of the legal limits at the time of reaching the predetermined best before date.

When those oils were also randomly tested from retail shelves under real life conditions, an even smaller proportion of oils failed one or more chemical and/or organoleptic limits for extra virgin olive oil according to most common international standards. 
Data collected from shelf testing would indicate that an additional time of 1 to 2 months may have to be deducted from the initial formula in order to compensate for the average exposure of the oils to less than ideal storage conditions during transport, handling, and presence on the shelves.

\section{Competing Interests}

The authors have no competing interests regarding the publication of the paper.

\section{References}

[1] R. Farhoosh and S.-Z. Hoseini-Yazdi, "Shelf-life prediction of olive oils using empirical models developed at low and high temperatures," Food Chemistry, vol. 141, no. 1, pp. 557-565, 2013.

[2] E. N. Frankel, Lipid Oxidation, The Oily Press, Dundee, Scotland, 1998.

[3] M.Z. Tsimidou, "Olive oil quality," in Chemistry and Technology, D. Boskou, Ed., chapter 6, AOCS, 2nd edition, 2006.

[4] R. Mateos, M. Trujillo, M. Carmen Pérez-Camino, W. Moreda, and A. Cert, "Relationships between oxidative stability, triacylglycerol composition, and antioxidant content in olive oil matrices," Journal of Agricultural and Food Chemistry, vol. 53, no. 14, pp. 5766-5771, 2005.

[5] A. Vazquez Roncero, C. Janer del Valle, and M. L. Janer del Valle, "Natural polyphenols and stability of olive oil," Grasas Aceites, vol. 26, pp. 14-18, 1975.

[6] M. Servili and G. Montedoro, "Contribution of phenolic compounds to virgin olive oil quality," European Journal of Lipid Science and Technology, vol. 104, no. 9-10, pp. 602-613, 2002.

[7] V. Mancebo-Campos, M. D. Salvador, and G. Fregapane, "Comparative study of virgin olive oil behavior under rancimat accelerated oxidation conditions and long-term room temperature storage," Journal of Agricultural and Food Chemistry, vol. 55, no. 20, pp. 8231-8236, 2007.

[8] E. Psomiadou, K. X. Karakostas, G. Blekas, M. Z. Tsimidou, and D. Boskou, "Proposed parameters for monitoring quality of virgin olive oil (Koroneiki cv)," European Journal of Lipid Science and Technology, vol. 105, no. 8, pp. 403-408, 2003.

[9] K. Hrncirik and S. Fritsche, "Relation between the endogenous antioxidant system and the quality of extra virgin olive oil under accelerated storage conditions," Journal of Agricultural and Food Chemistry, vol. 53, no. 6, pp. 2103-2110, 2005.

[10] E. Monteleone, G. Caporale, A. Carlucci, and E. Pagliarini, "Optimisation of extra virgin olive oil quality," Journal of the Science of Food and Agriculture, vol. 77, no. 1, pp. 31-37, 1998.

[11] E. Pagliarini, B. Zanoni, and G. Giovenelli, "Predictive study on Tuscan EVOO stability under several commercial conditions," Journal of Agricultural and Food Chemistry, vol. 48, pp. 145-1351, 2000.

[12] F. Gutiérrez and J. L. Fernández, "Determinant parameters and components in the storage of virgin olive oil. Prediction of storage time beyond which the oil is no longer of 'extra' quality," Journal of Agricultural and Food Chemistry, vol. 50, no. 3, pp. 571-577, 2002.

[13] F. A. Coutelieris and A. Kanavouras, "Experimental and theoretical investigation of packaged olive oil: development of a quality indicator based on mathematical predictions," Journal of Food Engineering, vol. 73, no. 1, pp. 85-92, 2006.
[14] E. N. Frankel, R. J. Mailer, S. C. Wang et al., Evaluation of ExtraVirgin Olive Oil Sold in California, UC Davis, Davis, Calif, USA, 2011.

[15] C. Guillaume and L. Ravetti, Evaluation of New Analytical Methods to Detect Lower Quality Olive Oils, Rural Industries Research and Development Corporation, Canberra, Australia, 2012.

[16] J. C. Moore, J. Spink, and M. Lipp, "Development and application of a database of food ingredient fraud and economically motivated adulteration from 1980 to 2010," Journal of Food Science, vol. 77, no. 4, pp. R118-R126, 2012.

[17] C. Guillaume, Ch. Gertz, and L. Ravetti, "Pyropheophytin a and 1,2-diacyl-glycerols over time under different storage conditions in natural olive oils," Journal of the American Oil Chemists' Society, vol. 91, no. 5, pp. 697-709, 2014. 

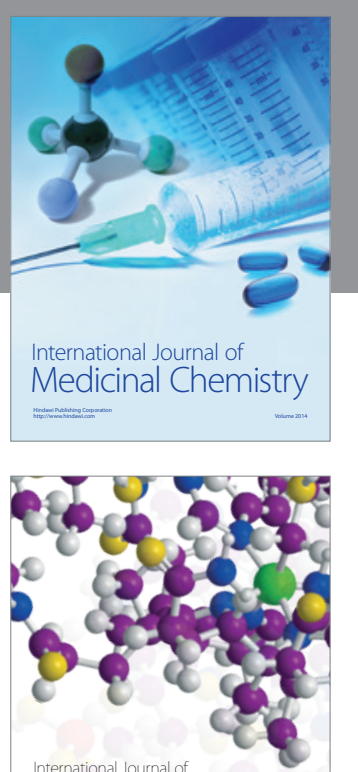

Carbohydrate Chemistry

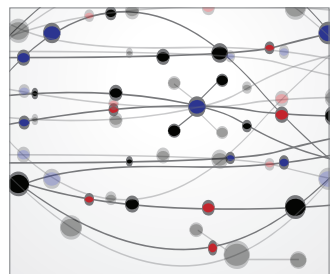

The Scientific World Journal
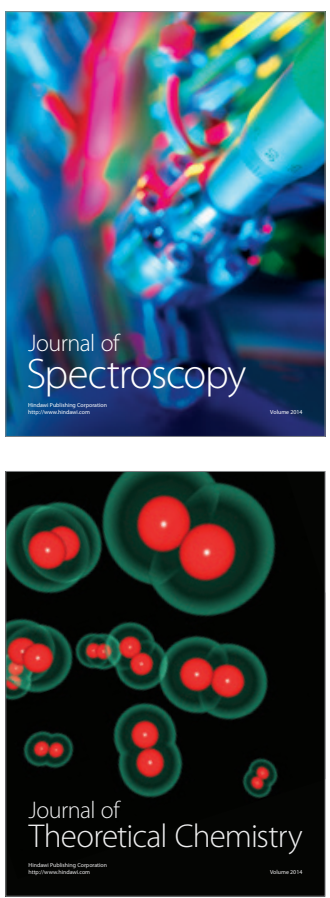
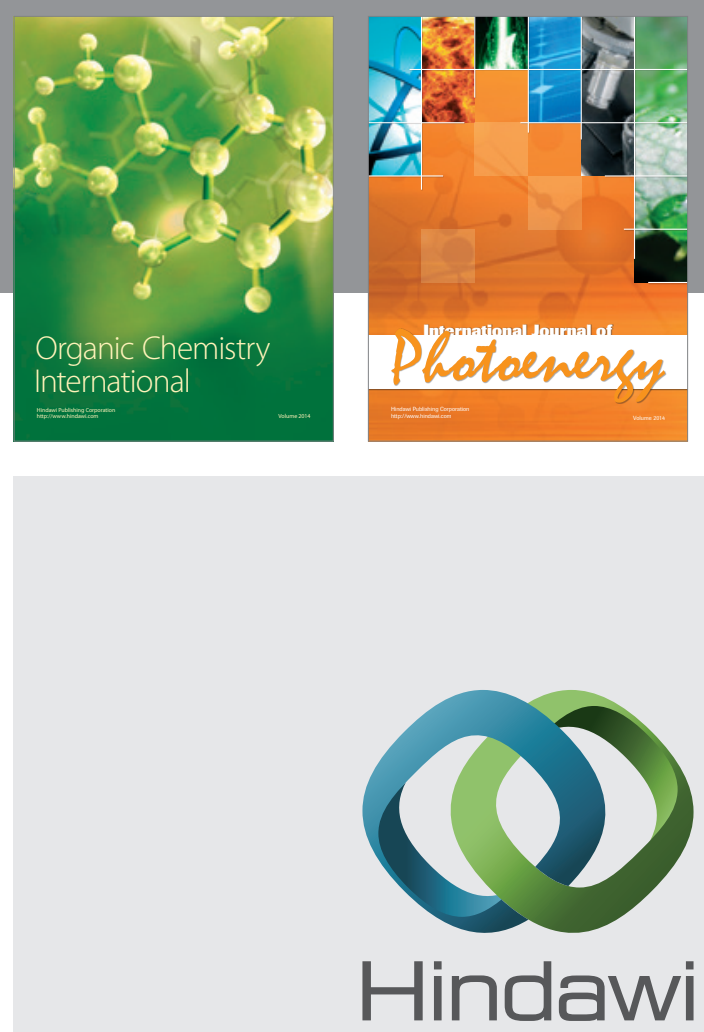

Submit your manuscripts at

http://www.hindawi.com

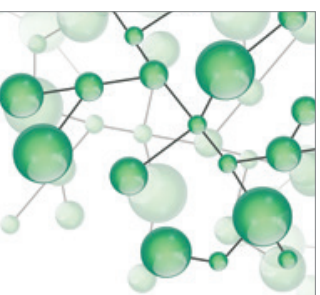

International Journal of

Inorganic Chemistry

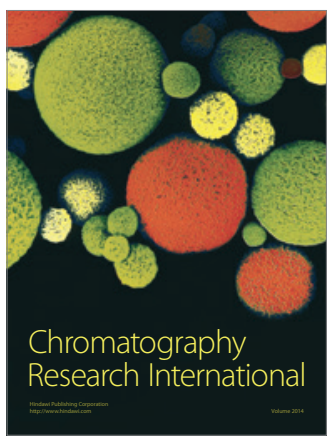

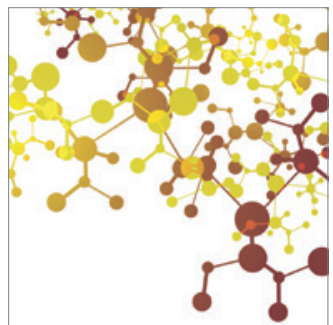

Applied Chemistry
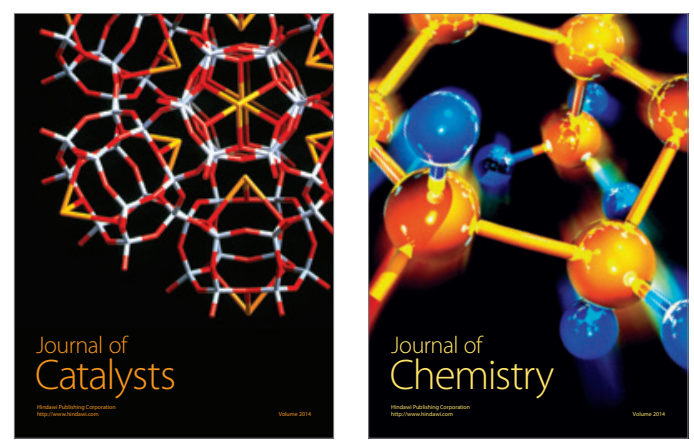
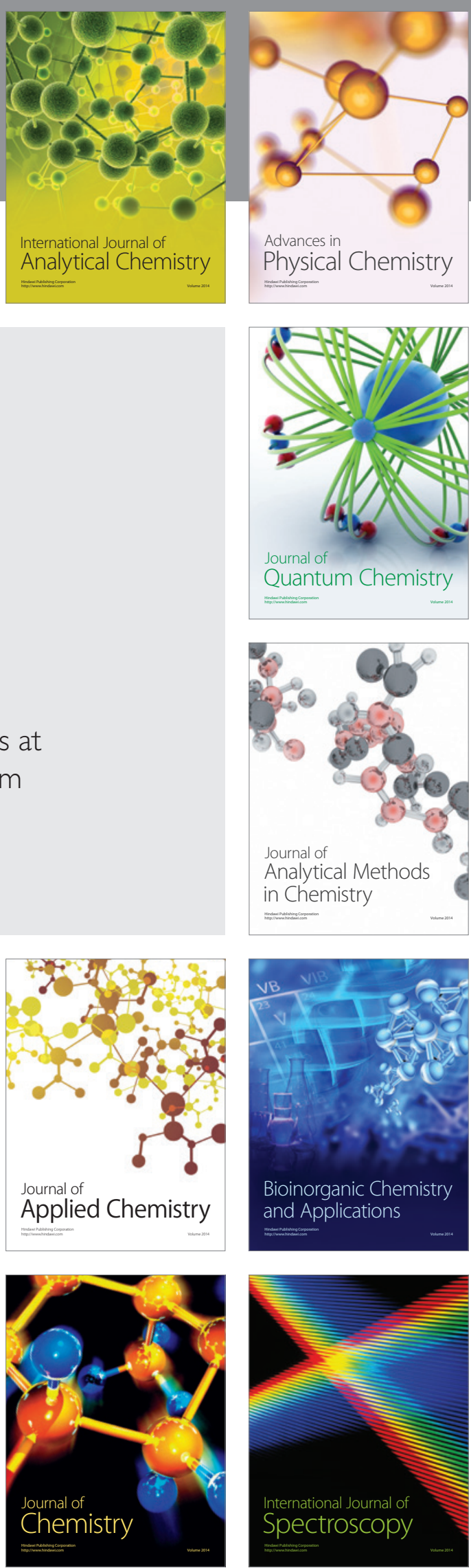